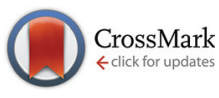

Cite this: Analyst, 2015, 140, 5012

Received 3rd May 2015,

Accepted 4th June 2015

DOI: $10.1039 / c 5 a n 00878 f$

www.rsc.org/analyst

\section{Quantitative enantioselective Raman spectroscopy $\dagger$}

\author{
J. Kiefer $r^{a, b, c}$
}

\begin{abstract}
Analytical methods for quantitative enantioselective measurements are highly desirable in the life sciences. Existing technologies have disadvantages such as limited temporal resolution, the need for molecular labeling, or high experimental complexity. To overcome these limitations, this work presents a method based on conventional Raman spectroscopy. A systematic investigation of the key parameters is carried out. It is demonstrated that their careful choice provides an opportunity for enantioselective and quantitative analysis of enantiopure systems as well as enantiomer mixtures.
\end{abstract}

Many biologically and pharmaceutically active molecules are chiral, ${ }^{1}$ for instance amino acids and sugars. Chemical synthesis of such compounds usually generates the racemate, which is a $1: 1$ mixture of both enantiomers. However, the enantiomers of a chiral substance may exhibit very different physiological effects. Prominent examples are the nonsteroidal anti-inflammatory drug ibuprofen and the immune-modulatory drug thalidomide. In case of ibuprofen, one enantiomer is the drug exhibiting the desired pharmaceutical activity, while its counterpart is virtually inactive. Hence, there is no need to purify one enantiomer as the racemate can be marketed. ${ }^{2}$ On the other hand, thalidomide is a completely different story: one enantiomer possesses the desired effect, while the other can cause birth defects in children. ${ }^{3}$ Therefore, it is important that such a chiral substance is produced with high enantiomeric purity.

The key to ensure enantiomeric purity is to have effective methods in place for monitoring the synthesis and purification process. A suitable analytical technology should facilitate speciation/molecular identification, enantiomeric

\footnotetext{
${ }^{a}$ Technische Thermodynamik, Universität Bremen, Badgasteiner Str. 1, 28359 Bremen, Germany.E-mail: jkiefer@uni-bremen.de

${ }^{b}$ School of Engineering, University of Aberdeen, Fraser Noble Building, Aberdeen AB24 3UE, UK

${ }^{c}$ Erlangen Graduate School in Advanced Optical Technologies, Universität ErlangenNürnberg, 91052 Erlangen, Germany

$\dagger$ Electronic supplementary information (ESI) available. See DOI: 10.1039/ C5AN00878F
}

discrimination, and quantification of the enantiomeric ratio. These requirements represent true challenges, as the enantiomers of a chiral substance possess identical physicochemical properties. Methods capable of differentiating enantiomers include NMR spectroscopy, ${ }^{4}$ microwave $^{5}$ and fluorescence ${ }^{6}$ spectroscopy, vibrational circular dichroism (VCD), ${ }^{7}$ and cavity ringdown polarimetry. ${ }^{8}$ However, all these methods have disadvantages. Some of them are time-consuming and hence do not provide sufficient time-resolution for process monitoring, some require molecular labeling or the addition of a chemical agent, and some are expensive and experimentally complicated.

Analytical techniques based on the Raman effect are interesting alternatives, as they have the potential to provide detailed and quantitative information about the chemical structure and composition. The most established tool in the context of chiral systems is Raman optical activity (ROA). ${ }^{9-11}$ In a ROA experiment, the sample is exposed to circularly polarized light and the difference intensity spectrum obtained from right- and left-handed circular irradiation yields enantioselective structural information. ${ }^{10} \mathrm{~A}$ drawback, however, can be the measurement time, which is usually in the order of minutes to hours; hence the potential for process monitoring is limited. Another Raman method is surface enhanced Raman spectroscopy (SERS), which has been demonstrated as a tool for enantiomeric discrimination recently. ${ }^{12}$ SERS utilizes the Raman signal enhancement in the close vicinity of a plasmonic material, e.g. a metal nanoparticle. When this material interacts with the target species in an enantioselective way, enantiomeric discrimination is possible. Disadvantages of SERS for process monitoring are that the plasmonic agent needs to be added. This may be expensive and not practicable. Furthermore, signal quantification is difficult. ${ }^{13}$ The disadvantages of both ROA and SERS can be overcome using conventional Raman spectroscopy with polarization-resolved signal detection. Raman scattering is frequently used as a tool for structural $^{14,15}$ and compositional ${ }^{16,17}$ analysis. However, enantioselective measurements were believed to be impossible in the past. This limitation has been overcome only recently. ${ }^{18}$ 
In a recent Communication, ${ }^{18}$ we have proposed a concept that allows the qualitative discrimination of the enantiomers of a chiral substance using Raman spectroscopy for the first time. For this purpose, a simple half-wave retarder was inserted in a polarization-resolved Raman setup and the polarization behavior of the laser and the Raman signal was exploited. ${ }^{19}$ The present article extends the qualitative enantioselective approach to facilitate quantitative measurements. Hence, it makes an important step towards using this new method in practical applications. For completeness, it should be pointed out that the proposed method utilizes the effect of optical activity on the Raman signal regardless of the origin of the optical activity. This means that enantioselective Raman spectroscopy can be used to directly monitor the Raman peaks of a chiral substance or to indirectly detect the chirality via the peaks of an achiral species in the sample, e.g. a solvent.

Quantitative measurements in a chiral system can have two main objectives: (1) to determine the overall concentration of a target species, and (2) to determine the concentration ratio of the enantiomers of the target species. In order to achieve these goals, the effects of all key parameters on the signal behavior must be understood for the proposed enantioselective Raman technique. The main experimental parameter to be taken into account is the rotation angle of the half-wave retarder. On the sample side, the Raman depolarization ratio is crucial. The effects of both need to be analyzed for varying enantiomer concentration. In the present work, these effects are studied computationally in order to avoid any bias from experimental artifacts. The influence of the parameters of interest on the signal polarization and intensity are modeled using first principles of physics. A detailed description of the method and the underlying mathematical framework are laid out in the $\mathrm{ESI}^{\dagger}$ together with a description of all the parameters. In the following, we analyze the effects of retarder orientation, Raman depolarization and concentration on a single isolated peak in the Raman spectrum.

The key component of the enantioselective Raman setup is the half-wave retarder. Hence, it is important to understand its performance in an experiment. The most important parameter is the angle between the optical axis of the retarder and the polarization direction of the light. A half-wave retarder rotates the polarization plane in such a way that it doubles this angle. This means that if the angle is $5^{\circ}$, for example, the polarization direction will be rotated by another $5^{\circ}$ so that the final angle between the optical axis and the transmitted light is $10^{\circ}$. In polarization-resolved Raman spectroscopy, this effect must be taken into account for both the vertically and horizontally polarized signal components. The angle $\psi$ is defined between the retarder optical axis and the $z$-axis of the coordinate system (see ESI for a sketch $\dagger$ ). The $z$-axis denotes the polarization direction of the initial laser beam propagating in $x$-direction. The angle, by which the Raman signal is rotated when travelling through the sample is $\phi$. Hence, the relevant angle with respect to the signal polarization exiting the sample is given by $\phi-\psi$, and the resulting

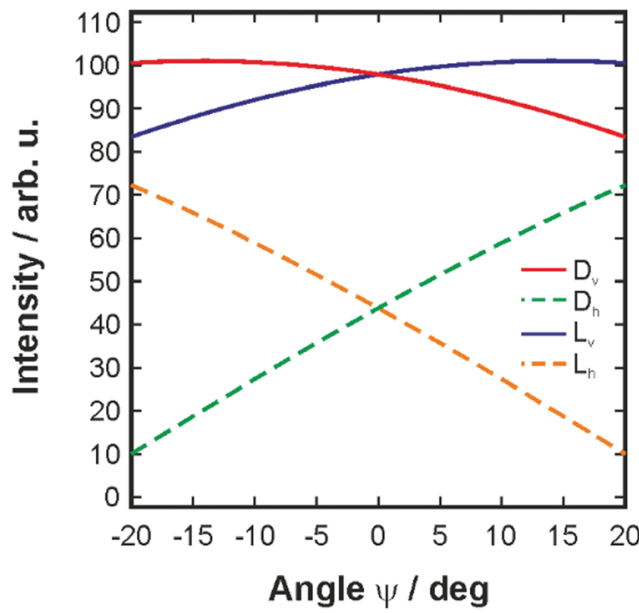

Fig. 1 Intensity of the vertically and horizontally polarized signal components in dependence on the half-wave plate orientation. In the legend, $\mathrm{D}$ and $\mathrm{L}$ indicate the enantiomer and the subscripts $\mathrm{v}$ and $\mathrm{h}$ correspond to the polarization.

signal polarization angle $\phi_{\text {new }}$ with respect to the $z$-axis becomes

$$
\phi_{\text {new }}=2(\phi-\psi)+\psi=2 \phi-\psi
$$

With this new angle, the intensity projections can be calculated using eqn (S1) and (S2) in the ESI† yielding the intensities of the detected vertically and horizontally polarized signals. Fig. 1 displays the intensities of the vertically and horizontally polarized signal components as function of the rotation angle of the half-wave retarder. The data have been simulated assuming a constant depolarization ratio of 0.2 and the maximum concentration of 1 for one or the other enantiomer. The latter means absolute values for $\theta$ and $\phi$ of $5^{\circ}$ and $10^{\circ}$, respectively, where the D-enantiomer will exhibit a negative sign. The angle $\theta$ is the angle by which the laser polarization is rotated at the measurement point in the sample with respect to the $z$-axis.

The angle between the optical axis of the half-wave plate and the $z$-axis has been varied from $-20^{\circ}$ to $+20^{\circ}$, in other words $-2 \phi$ to $+2 \phi$. For the $\psi=0^{\circ}$, the signals of both enantiomers are rotated by the same angle in terms of absolute value, and the remaining symmetry of the polarization diagram results in identical vertical and horizontal signal components for both enantiomers. The other extreme values considered are $-20^{\circ}$ and $+20^{\circ}$. In both cases, the polarized signal component, $I_{\text {pol }}$, of one of the enantiomers is rotated such that it coincides with the $z$-axis, and the other one ends up at $+40^{\circ}$ or $-40^{\circ}$, respectively. This situation represents the highest possible asymmetry; hence, the largest differences in signal intensities and the best discrimination of the enantiomers. However, it must be kept in mind that the data displayed correspond to one specific Raman depolarization ratio and a given concentration. In the full Raman spectrum of a chiral molecule, there will be numerous peaks exhibiting different depolarization 
ratios, and the concentration may be unknown. Consequently, selecting an angle $\psi$ for an experiment may be not as straightforward as Fig. 1 would suggest at first glance. Generally speaking, any angle that does not equal zero works in principle, but a test/calibration measurement using a defined sample is helpful to optimize the alignment.

As previously mentioned, the Raman spectrum of a chiral substance comprises peaks exhibiting different depolarization ratio. Consequently, different peaks will result in different polarization-resolved signal intensities. Moreover, these signal intensities will depend on the orientation angle of the halfwave-retarder.

The Raman depolarization ratio $\rho$ is defined as the ratio of the depolarized and the polarized intensities

$$
\rho=\frac{I_{\mathrm{depol}}}{I_{\mathrm{pol}}}
$$

and it provides useful information about the symmetry of a vibrational mode. $^{20-22}$ Its values lie in the range between $\sim 0$ for highly polarized lines and 0.75 for completely depolarized lines. ${ }^{23}$ The combined effect of varying the depolarization ratio and the retarder orientation is illustrated in Fig. 2, which shows the detected vertical and horizontal signal intensities for both enantiomers. The angle $\psi$ has been varied between $-20^{\circ}$ and $0^{\circ}$ only, as the corresponding positive values would result in analog, mirrored signals for both enantiomers.

The surfaces plotted for the L-enantiomer (lower panels) exhibit an almost negligible curvature (it is not zero though). This is reasonable, because for the considered values of $\psi$, the resulting rotation angle $\phi_{\text {new }}$ varies between $+40^{\circ}$ and $+20^{\circ}$, a range in which sine and cosine functions do not possess severe nonlinearities. On the other hand, the intensity surfaces of the D-enantiomer exhibit significant curvature, as the rotation angle $\phi_{\text {new }}$ varies between $0^{\circ}$ and $-20^{\circ}$. In particular, the cosine is highly nonlinear in this range.
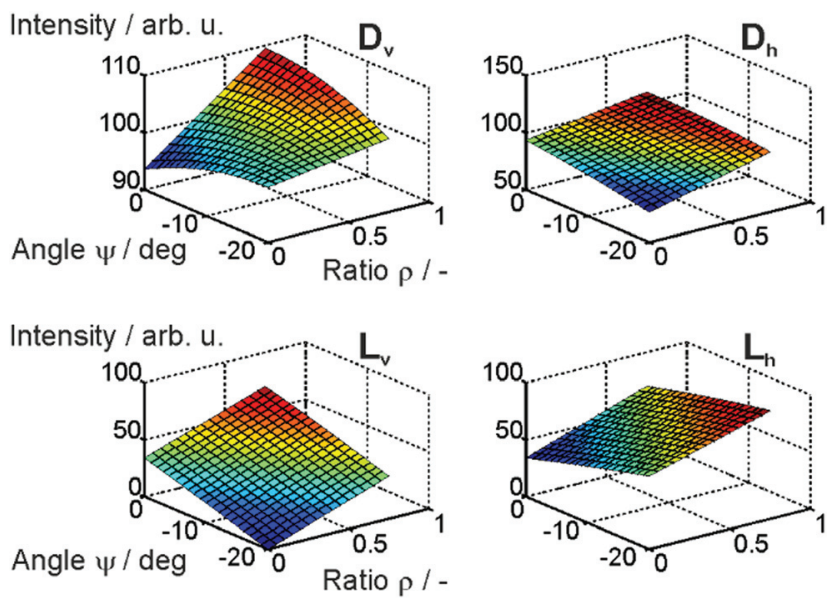

Fig. 2 Intensity of the vertically and horizontally polarized signal components in dependence on the Raman depolarization ratio and the halfwave plate orientation. In the legend, D and L indicate the enantiomer and the subscripts $\mathrm{v}$ and $\mathrm{h}$ correspond to the polarization.
The main objective in quantitative measurements is to determine the concentration of the target species, and hence the influence of the concentration on the signal needs to be looked at. In the following, we consider samples containing one enantiomer only, and thereafter samples comprising both enantiomers in different ratios will be discussed.

The enantiomer concentration $c$ exerts an influence on the measured signal in two different ways. First, the signal intensity is a function of concentration, $I(c)$. As a reasonable approximation, this function can be assumed linear: doubling the number of probed molecules will increase the signal intensity by a factor of two. Note that molecular interactions can lead to peak shifts and changes in the polarizability of a vibrational mode, ${ }^{24}$ and hence lead to nonlinearities when the signal at a given wavenumber is monitored as a function of concentration. Such effects are neglected here. Introducing a normalized concentration of the target species, which varies between 0 and 1 , the concentration dependence of the initial polarized and depolarized components is given as

$$
I_{\mathrm{pol} / \mathrm{depol}}=c I(c=1) \text {. }
$$

In our example, $I_{\text {pol }}(c=1)$ is 100 and $I_{\text {depol }}(c=1)$ is 20 to yield the depolarization ratio of 0.2 for consistency with previous data.

Second, the influence of the concentration on the optical activity of the sample must be taken into account. The specific rotation $\alpha$ is normally proportional to the concentration and given as

$$
\alpha=\alpha_{\lambda}^{T} c x
$$

where $\alpha_{\lambda}^{T}$ is a species-specific constant depending on temperature $T$ and wavelength $\lambda$, and $x$ is the distance, which the light travels inside the sample. This means that $\theta$ and $\phi$ become functions of concentration.

The resulting concentration effects on the detected vertically and horizontally polarized signal components are illustrated in the upper and center row panels of Fig. 3. Again, a variation of the retarder orientation is considered as well. For $\psi=0^{\circ}$, both enantiomers show identical behavior, while distinct differences can be found when the angle is varied. The nonlinearities are more pronounced in the signals with horizontal polarization as they exhibit generally low intensity and therefore, small changes in the conditions in terms of concentration and angle and their interplay result in significant alterations, while the vertical components appear almost unaffected by the retarder orientation $\psi$. Interestingly, the horizontally polarized signal of the L-enantiomer shows a line of local minima between $c=0 / \psi=0^{\circ}$ and $c=1 / \psi=-20^{\circ}$. This line connects all those points at which the retarder rotates the polarization such that it coincides with the $z$-axis; in other words, the angle $\phi_{\text {new }}$ becomes zero. The panels of the lower row of Fig. 3 display the difference intensities of the vertically and horizontally polarized signal components for the $\mathrm{D}^{-}$and L-enantiomer. Note that the $\psi$-axis is plotted with reversed direction compared to the other graphs in order to 

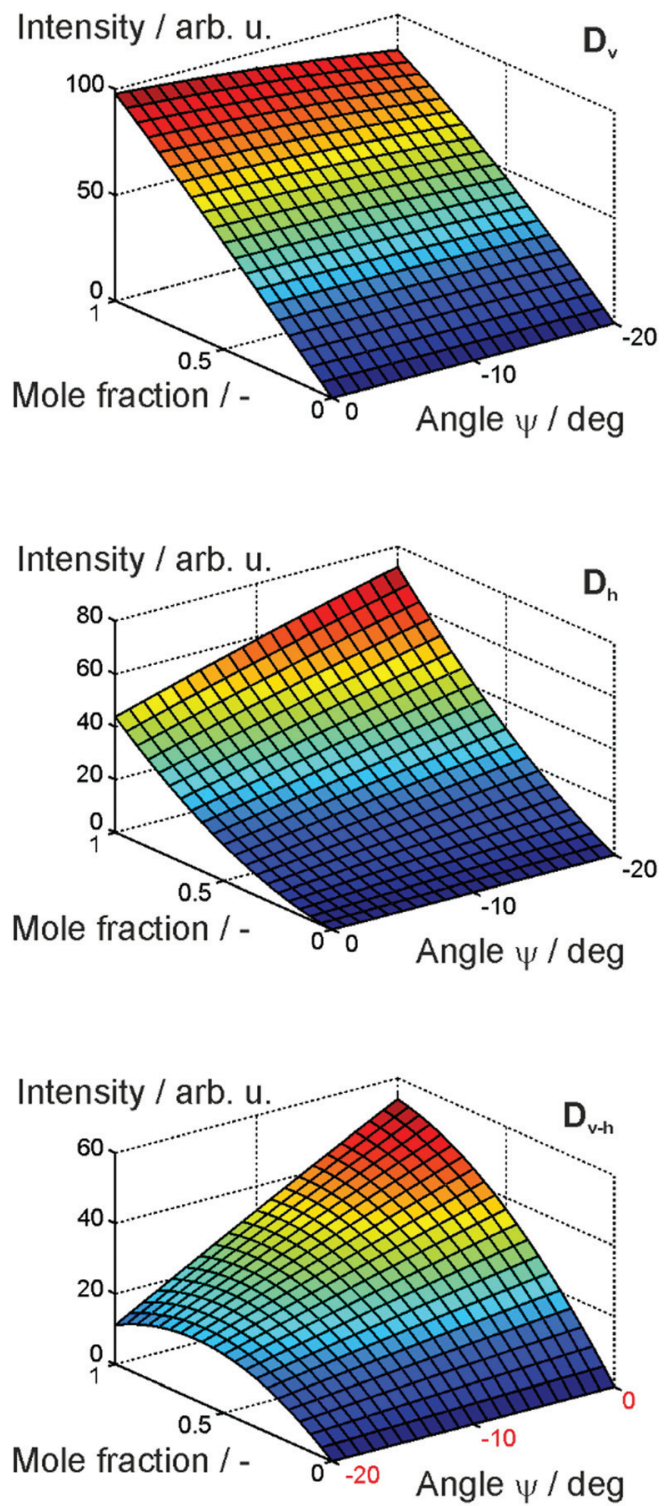
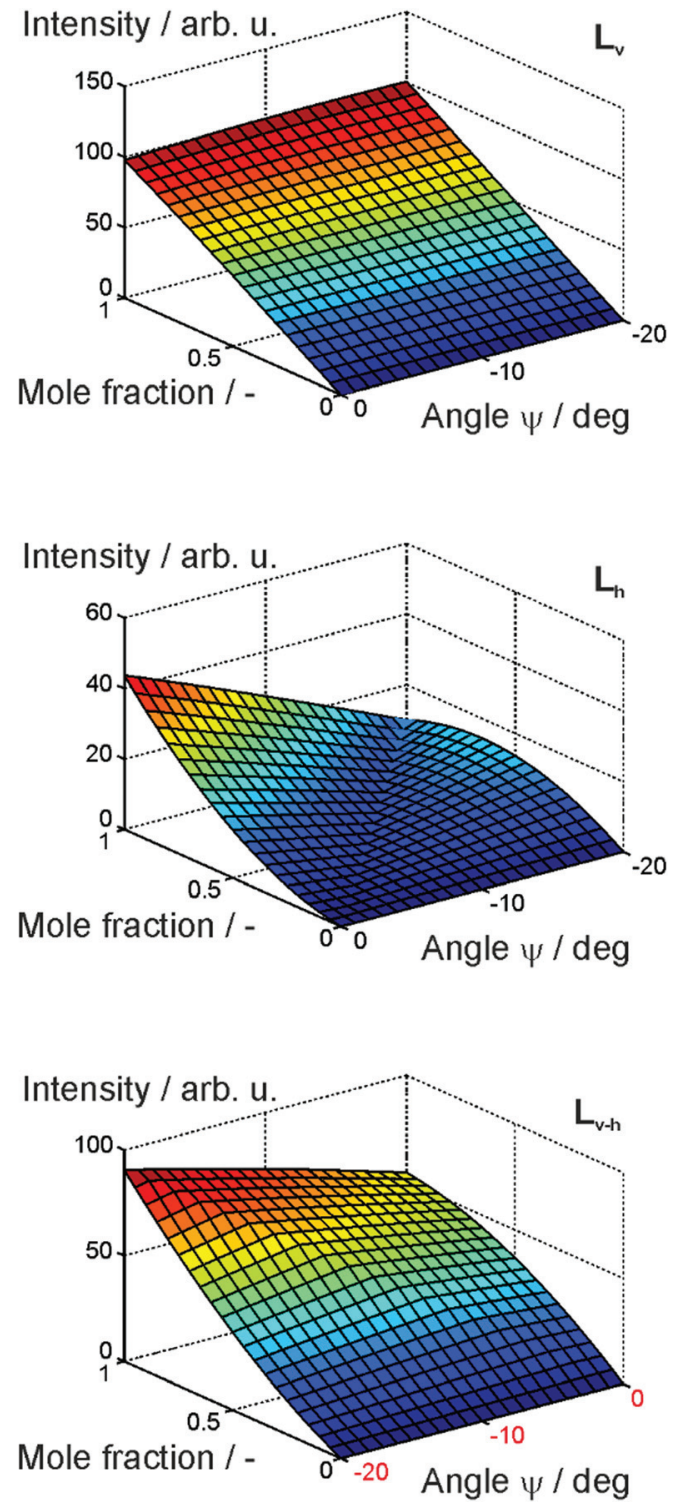

Fig. 3 Intensity of the vertically (upper row) and horizontally (center row) polarized signal components in dependence on the concentration and the half-wave plate orientation. The panels in the lower row represent the difference of vertical and horizontal component. $D$ and $L$ indicate the enantiomer and the subscripts $\mathrm{v}$ and $\mathrm{h}$ correspond to the polarization state.

improve readability (otherwise, parts of the surfaces would be hardly visible or obscured). The curvature is dominated by the horizontally polarized signal. It becomes clear that both enantiomers exhibit significantly different behavior and hence can be distinguished using the difference signal.

Chemical synthesis usually generates the racemate, which is a 1:1 mixture of both enantiomers. Consequently, the production and downstream processing steps will deal with mixtures containing both enantiomers of a chiral substance. Therefore, in most practical situations, the analytical task will be to perform measurements in such a mixture. In the following, the effects of enantiomer mixtures of systematically varied composition will be analyzed. Common parameters for charac- terizing mixtures of enantiomers are the enantiomeric ratio er, the enantiomer excess ee and the optical purity op. ${ }^{25}$ The enantiomeric ratio is the ratio of the percentage of one enantiomer in a mixture to that of the other; it is normally defined such that it takes values of 1 (for the racemate) and above. The enantiomer excess is the difference of both percentages, and the optical purity represents the ratio of the observed optical rotation of a sample consisting of a mixture of enantiomers to the optical rotation of one pure enantiomer. However, these parameters are not always appropriate for characterizing a sample and, hence, we stick to plain concentration values in the following. Again, normalized concentrations taking values between 0 and 1 that can be interpreted in terms of molar fraction are used. 
Above, the concentration effects for the case of having only one enantiomer have been discussed. In a mixture, however, both compounds have to be taken into account. For the concentration dependence of the intensity, this means that the intensities must be added to yield

$$
I_{\text {pol } / \text { depol }}^{\mathrm{D}+\mathrm{L}}=c_{\mathrm{D}} I^{\mathrm{D}}\left(c_{\mathrm{D}}=1\right)+c_{\mathrm{L}} I^{\mathrm{L}}\left(c_{\mathrm{L}}=1\right)=\left(c_{\mathrm{D}}+c_{\mathrm{L}}\right) I(c=1) .
$$

The simplification on the RHS of eqn (5) is possible as the Raman scattering cross section of a given vibration is identical for both enantiomers. The change in the specific rotation can be described according to

$$
\alpha^{\mathrm{D}+\mathrm{L}}=\alpha_{\lambda}^{T, \mathrm{D}} x\left(c_{\mathrm{D}}-c_{\mathrm{L}}\right)
$$

(6) from measured signals can be illustrated using plots of the where $\alpha_{\lambda}^{T, \mathrm{D}}$ is the rotation constant of the D-enantio mer.

Fig. 4 illustrates the detected signal intensities as function of the mixture composition. The four rows show data obtained for four different retarder orientation angles $\psi$. For $\psi=0^{\circ}$, the diagrams are perfectly symmetric with respect to a plane parallel to the intensity axis and containing the equimolar line, i.e. $c_{\mathrm{L}}=c_{\mathrm{D}}$. In this case, it is possible to determine the overall content of the chiral species but not its enantiomer ratio. With increasing angle $\psi$, the apparent symmetry plane is shifted and disappears completely for $\psi=-20^{\circ}$. Hence, it is possible to determine the mixture composition unambiguously.

A straightforward way of deriving quantitative information
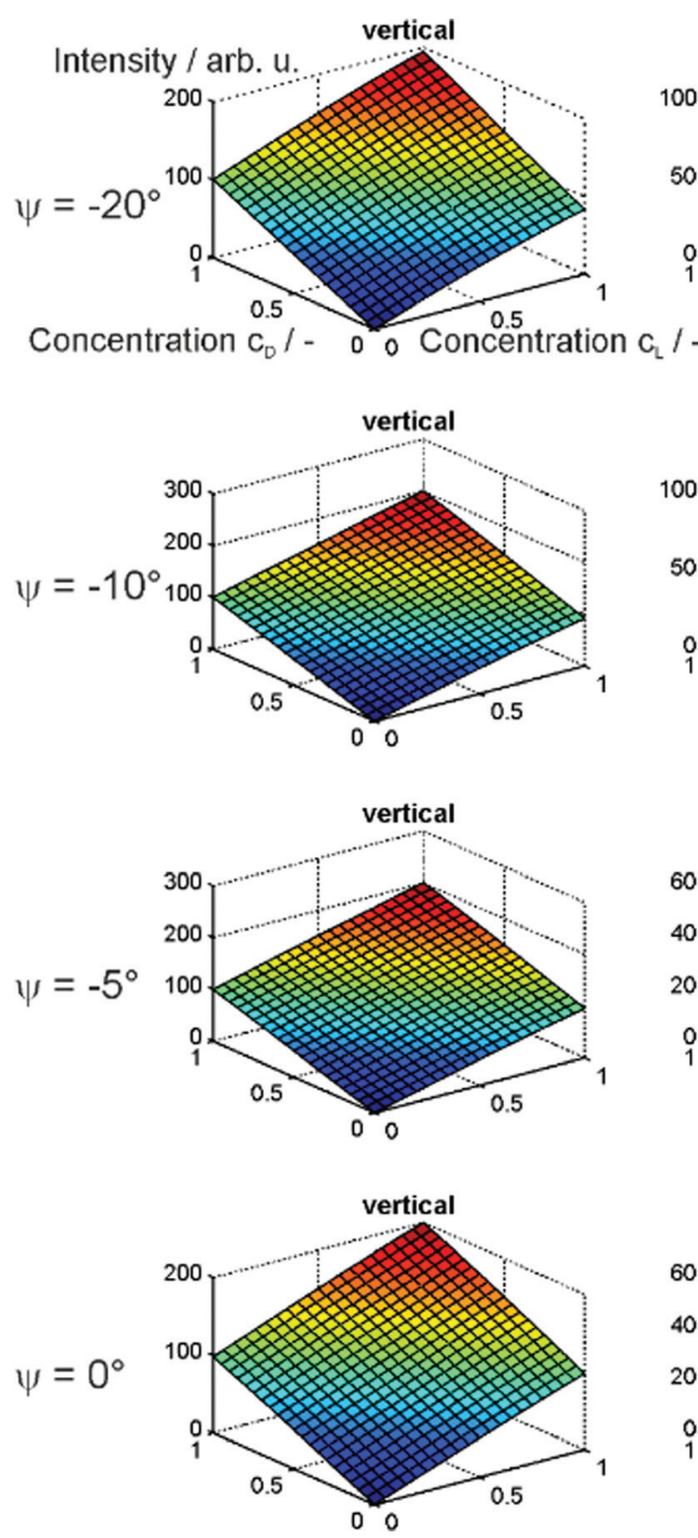
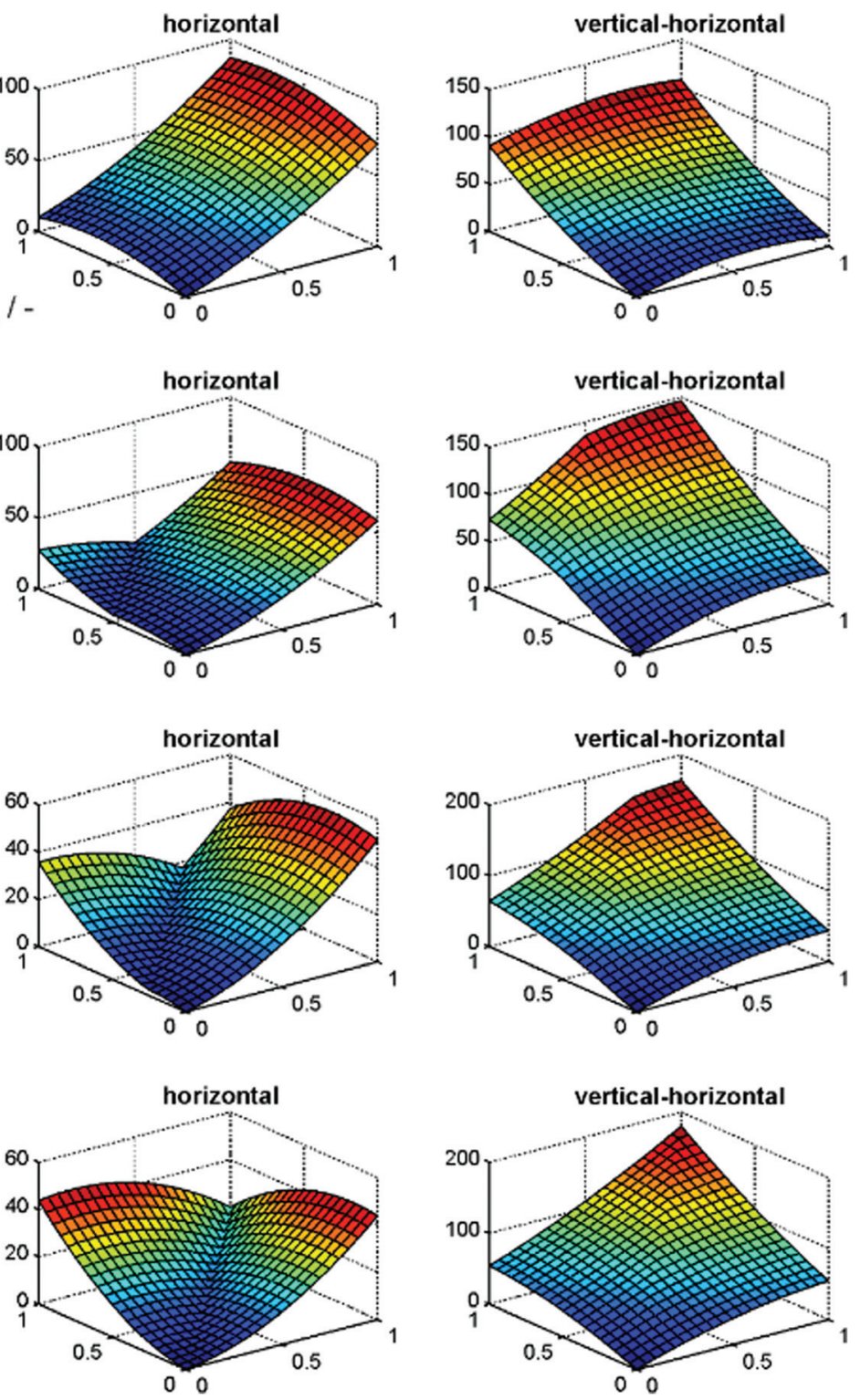

Fig. 4 Intensity of the vertically (first column) and horizontally (second column) polarized signal components, and their difference (third column) in dependence on the enantiomer concentrations. The panels in the different rows illustrate the influence of the half-wave plate orientation. 

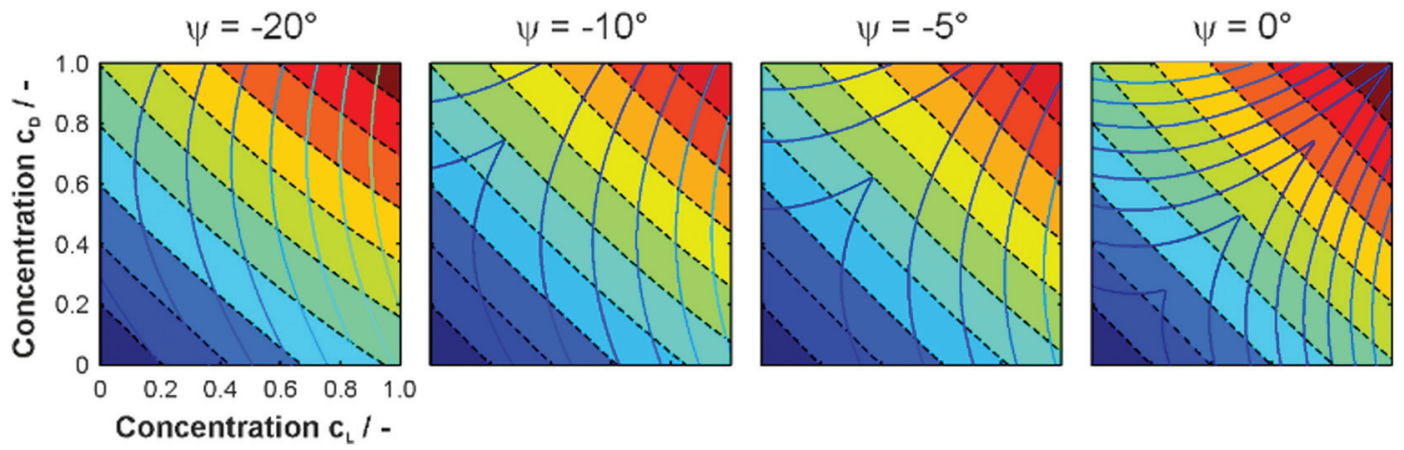

Fig. 5 Iso-intensity contour lines of the vertically (dashed lines) and horizontally (solid lines) polarized signal components for four different halfwave plate orientation angles.

contour lines of the surfaces in Fig. 4. Fig. 5 shows these plots for the four different cases. The solid lines correspond to the horizontally polarized signal component and the dashed lines and colored areas to its vertical counterpart. For a given (measured) intensity, a corresponding line can be found for either signal polarization. Those lines connect all the concentration pairs $c_{\mathrm{L}} / c_{\mathrm{D}}$ that would lead to the same signal intensity. As the lines exhibit different behavior for both polarizations, their points of intersection give the possible concentration pairs $c_{\mathrm{L}} / c_{\mathrm{D}}$. For $\psi=-20^{\circ}$, there is a single, unique point of intersection enabling unambiguous determination of the enantiomeric composition. For smaller angles, there can be two possible pairs of concentrations and hence care must be taken. A rather simple method to avoid this situation is to do an initial calibration measurement in a sample containing the highest expected concentration of one enantiomer, and subsequently adjusting the half-wave retarder such that $\psi=2 \phi$. Estimating $\phi$ from literature data of the specific rotation is a suitable alternative, see for example ref. 26.

\section{Conclusions}

The potential of using enantioselective Raman spectroscopy for quantitative measurements has been investigated. The influence of the main factors, i.e. the Raman depolarization ratio, the orientation angle of the half-wave retarder, and the enantiomer concentration, have been analyzed. From the results, the following conclusions and recommendations can be made:

- When discrimination of the enantiomers is the only task, looking at either the vertically or the horizontally polarized signal component is in principal sufficient. The difference spectrum viz. vertical minus horizontal component is a suitable and likely more sensitive alternative. It should be noted that the enantioselective Raman method does not just provide information about the sign of the rotation angle like in optical rotation measurements. The Raman spectra of both sample cases (positive and negative sign) are different such that they exhibit the same peaks at the same spectral positions, but their relative intensities are different; hence, the Raman spectrum of an optically active solution is unique with the proposed method.

- It should be pointed out that the enantioselective Raman approach utilizes the optical activity regardless of its origin. Hence, it allows monitoring the peaks of a chiral substance directly or studying the Raman signal of an achiral solvent, for example. In both cases, enantioselective discrimination is possible. This is a significant difference in comparison to Raman Optical Activity measurements, from which a complete molecule-specific three-dimensional solution structure can be obtained. ${ }^{27}$

- In enantiopure samples, the orientation angle $\psi$ can in principle be chosen arbitrarily. As long as it is different from zero, the setup will allow the discrimination of the enantiomers and determination of the concentration. The optimal angle was found to be $\psi= \pm 2 \phi$, where $\phi$ is the angle by which the signal polarization is rotated in the sample with the highest possible enantiomer concentration, as it provides the most distinct difference between both enantiomers. The angle $\phi$ can be determined in a calibration measurement or it can be estimated utilizing values of the specific rotation of the target species from the literature.

- When the sample contains a mixture of both enantiomers, orientation angles $-2 \phi<\psi<+2 \phi$ may result in ambiguous signals. This means that two pairs of enantiomer concentrations can be found for a set of measured data. For $\psi=0^{\circ}$, there are always two concentration pairs of identical values with exchanged species. In other words, it is not possible to determine whether the $\mathrm{D}: \mathrm{L}$ mixture is $30: 70$ or $70: 30$, for example. Adjusting the orientation angle to $\psi= \pm 2 \phi$ yields unambiguous results.

In conclusion, the results demonstrate that enantioselective Raman spectroscopy can be a quantitative method for measuring enantiomer concentration and enantiomer ratio. These capabilities make Raman spectroscopy a unique tool for monitoring chiral systems of practical interest, for example, during the production and purification of pharmaceutically active ingredients. The method provides simultaneous structural and compositional analysis like conventional Raman spectroscopy combined with enantiomeric selectivity. Further key features 
of the proposed approach are short measurement times and high repetition rates; hence, it facilitates real-time process monitoring in chiral systems at reasonable costs. Therefore, the method can be a complement to established chiroptical techniques and a useful tool for fast initial screening of chiral samples.

\section{Notes and references}

1 R. E. Morris and X. H. Bu, Nat. Chem., 2010, 2, 353-361.

2 P. O. Carvalho, Q. B. Cass, S. A. Calafatti, F. J. Contesini and R. Bizaco, Brazilian J. Chem. Eng., 2006, 23, 291-300.

3 M. Emanuel, M. Rawlins, G. Duff and A. Breckenridge, Lancet, 2012, 380, 781-783.

4 D. Parker, Chem. Rev., 1991, 91, 1441-1457.

5 D. Patterson, M. Schnell and J. M. Doyle, Nature, 2013, 497, 475.

6 T. D. James, K. Sandanayake and S. Shinkai, Nature, 1995, 374, 345-347.

7 P. J. Stephens, F. J. Devlin and J. J. Pan, Chirality, 2008, 20, 643-663.

8 D. Sofikitis, L. Bougas, G. E. Katsoprinakis, A. K. Spiliotis, B. Loppinet and T. P. Rakitzis, Nature, 2014, 514, 76-79.

9 L. D. Barron, L. Hecht, E. W. Blanch and A. F. Bell, Prog. Biophys. Mol. Biol., 2000, 73, 1-49.

10 S. T. Mutter, F. Zielinski, P. L. A. Popelier and E. W. Blanch, Analyst, 2015, 140, 2944-2956.

11 L. A. Nafie, B. E. Brinson, X. L. Cao, D. A. Rice, O. M. Rahim, R. K. Dukor and N. J. Halas, Appl. Spectrosc., 2007, 61, 1103-1106.

12 Y. Wang, Z. Yu, W. Ji, Y. Tanaka, H. Sui, B. Zhao and Y. Ozaki, Angew. Chem., Int. Ed., 2014, 53, 13866-13870.
13 M. Sackmann and A. Materny, J. Raman Spectrosc., 2006, 37, 305-310.

14 J. De Gelder, K. De Gussem, P. Vandenabeele and L. Moens, J. Raman Spectrosc., 2007, 38, 1133-1147.

15 M. Sánchez del Rio, M. Picquart, E. Haro-Poniatowski, E. van Elslande and V. H. Uc, J. Raman Spectrosc., 2006, 37, 1046-1053.

16 K. Noack, B. Eskofier, J. Kiefer, C. Dilk, G. Bilow, M. Schirmer, R. Buchholz and A. Leipertz, Analyst, 2013, 138, 5639-5646.

17 J. Müller, K. Knop, J. Thies, C. Uerpmann and P. Kleinebudde, Drug Dev. Ind. Pharm., 2010, 36, 234-243.

18 J. Kiefer and K. Noack, Analyst, 2015, 140, 1787-1790.

19 J. Kiefer and M. Kaspereit, Anal. Methods, 2013, 5, 797-800.

20 R. W. Berg, J. N. Canongia Lopes, R. Ferreira, L. P. N. Rebelo, K. R. Seddon and A. A. Tomaszowska, J. Phys. Chem. A, 2010, 114, 10834-10841.

21 J. Kiefer, J. Fries and A. Leipertz, Appl. Spectrosc., 2007, 61, 1306-1311.

22 S. Hassing, K. D. Jernshoj and M. Hedegaard, J. Raman Spectrosc., 2011, 42, 21-35.

23 C. D. Allemand, Appl. Spectrosc., 1970, 24, 348-353.

24 K. Noack, A. Leipertz and J. Kiefer, J. Mol. Struct., 2012, 1018, 45-53.

25 A. D. McNaught and A. Wilkinson, IUPAC. Compendium of Chemical Terminology (the "Gold Book"); on-line corrected version created by M. Nic, J. Jirat, B. Kosata; updates compiled by A. Jenkins, Blackwell Scientific Publications, Oxford, 2nd edn, 1997.

26 R. C. Weast, Handbook of Chemistry and Physics, CRC Press, 55th edn, 1974.

27 V. Parchansky, J. Kapitan and P. Bour, RSC Adv., 2014, 4, 57125-57136. 